\title{
Threshold exposure duration for recognition of test objects in pre-school and school children
}

\begin{abstract}
Threshold exposure duration for recognition of test objects (TEDRTO) is minimum time required for recognition of the test object subtending a specified visual angle. 416 children of 5 to 17 years were investigated with special electronic apparatus. It was showed that the mean TEDRTO value decreases with age, from $2.1 \pm 1.3 \mathrm{~ms}$ in children of 5 to 6 years to $1.1 \pm 0.3 \mathrm{~ms}$ in children of 11 to 14 years and in children of 15 to 17 years. Therefore, TEDRTO variability diminishes with maturation of the visual system.
\end{abstract}

Volume 7 Issue 7 - 2017

\author{
Serdiuchenko VI, Zheliznyk MB \\ The Filatov Institute of Eye Diseases and Tissue Therapy NAMS \\ of Ukraine, Ukraine
}

\begin{abstract}
Correspondence: Serdiuchenko VI, The Filatov Institute of Eye Diseases and Tissue Therapy NAMS of Ukraine, Ukraine, Odessa,Ukraine, Email virais@ukr.net
\end{abstract}

Received: November 25, 2017| Published: December 18, 2017

\section{Actuality}

Threshold exposure duration for recognition of test objects (TEDRTO) has been assessed earlier in adults in ophthalmic ergonomic investigations. ${ }^{1-4}$ and in children with various refractive statuses, strabismus, and amblyopia., ${ }^{5,6}$ Investigation of age-related changes in TEDRTO in pre-school and school children with normal visual acuity is deemed to be important.

\section{Purpose}

The purpose study was to investigate threshold exposure duration for recognition of test objects in children of various age groups with a visual acuity of not less than 1.0 and without ophthalmic pathology.

\section{Materials and methods}

Four hundred and sixteen children of 5 to 17 years ( 5 to 7 years, 79 children; 7 to 10 years, 79 children; 11 to 14 years, 171 children; 15 to 17 years, 87 children) were included into the study. Other inclusion criteria were: no refracting medium pathology or fundus pathology and a refractive error within the normal range for the child's age (emmetropia or hyperopia of up to $1.0 \mathrm{D}$ ). Threshold exposure duration for recognition of test objects was assessed with a special electronic apparatus. The apparatus was used to expose a test object (an illuminated Landolt's ring with a gap randomly at one of the eight positions; ring luminance, 25 candela $/ \mathrm{m}^{2}$ ) against a black background, with the test object presented for $1 \mathrm{~ms}$ to $15 \mathrm{~ms}$, and $1 \mathrm{~ms}$ gaps between presentations. If the child failed to identify the target gap direction with the minimum exposure time, the time was increased until he could identify the direction fairly reliably, with no less than 5 correct identifications in succession. The test object had an angular size of 8 minutes of arc. Therefore, its image was projected onto the central fovea. Both monocular and binocular TEDRTO examinations were performed in the morning hours under photopic conditions. Due to the features of the data obtained for statistical analyses, we used the Kruskal-Wallis test, which is a non-parametric equivalent of analysis of variance, and extends the Mann-Whitney $\mathrm{U}$ test when there are $\mathrm{k}$ > 2 independent samples.

\section{Results}

Analysis of data showed that the children of age group 1 required statistically significantly more time for binocular recognition of white test objects than the children of other age groups. In addition, the mean TEDRTO value in age group 2 was greater than those in the older age groups. No difference in TEDRTO values between group 3 and group 4 was observed $(P=1.0)$. Similar relationships were observed for the TEDRTO related to monocular recognition of test objects. The older the children were, the less time their visual system required for the recognition of the test object presented. A similar relationship is seen for the use of a parametric statistical method. The mean TEDRTO value decreases with age, in binocular investigation from $2.1 \pm 1.3 \mathrm{~ms}$ in children of 5 to 6 years to $1,4 \pm 0,5 \mathrm{~ms}$ in children 7 to 10 years, $1.1 \pm 0.3 \mathrm{~ms}$ in children of 11 to 14 years and in children of 15 to 17 years.

Therefore, TEDRTO variability diminishes with maturation of the visual system: by the age of 11 years, TEDRTO values become stable, with no further changes. For comparison: higher TEDRTO values were received earlier in hyperopia $1.5-4.5$ dptr (14.9 \pm 1.3 $\mathrm{ms}$ and $11.8 \pm 1.1 \mathrm{~ms}$ in monocular and binocular investigations, respectively), which can be related to participation of accommodation in mechanism of test object recognition. ${ }^{6}$ TEDRTO values were even higher in anisometropic and disbinocular amblyopia $(50.5 \pm 8.6 \mathrm{~ms}$ and $112.5 \pm 15.8 \mathrm{~ms}$, respectively), which give an evidence of a significant slowing of test object recognition process in comparison with children with normal visual acuity. ${ }^{5}$

\section{Conclusion}

The results obtained can be used as reference values while investigating threshold exposure durations for recognition of test objects in various disorders of the visual system.

\section{Acknowledgments}

None. 


\section{Conflicts of interest}

Author declares that there are no conflicts of interest.

\section{Funding}

None.

\section{References}

1. Cobb PW. Some experiments on the speed of vision. Trans Illum Eng Soc. 1928;2(19):150-175.

2. Ferry C, Rand G. Intensity of light and speed of vision studied with the special reference to industrial situation. Part 2. Trans Illum Eng Soc. $1928 ; 23: 827$
3. Kolbanov VV, Medvedev VI. Dynamic characteristics of visual functions. Hum Physiol. 1979;4(5):687-693.

4. Neymark GS. The device for investigation of visual perception // Patent N 995734 (USSR). A 61 B 3/00; A 61 B 5/16. Publ 15.02.1993. Bull. N 6. 1993.

5. Serdiuchenko VI. Timing parameters of the resolving ability of the eye and their importance for the diagnosis and treatment of strabismus and amblyopia. Oftalmol Zh. 1994;5:262-265.

6. Serdiuchenko VI, Zheliznyk MB. Threshold exposure duration for recognition of test objects in children with various refractive statuses. Oftalmol Zh. 2016;3:3-5. 